Annals of Pure and Applied Mathematics

Vol. 16, No. 2, 2018, 337-343

ISSN: 2279-087X (P), 2279-0888(online)

Published on 22 February 2018

Annals of

www.researchmathsci.org

DOI: http://dx.doi.org/10.22457/apam.v16n2a10

Pure and Applied

Mathematics

\title{
Multiplicative Connectivity Revan Indices of Polycyclic Aromatic Hydrocarbons and Benzenoid Systems
}

\author{
V.R.Kulli \\ Department of Mathematics \\ Gulbarga University, Gulbarga 585106, India \\ e-mail:vrkulli@gmail.com
}

Received 9 February 2018; accepted 17 February 2018

\begin{abstract}
A chemical graph is a simple graph related to the structure of a chemical compound. The connectivity indices are applied to measure the chemical characteristics of compounds in Chemical Graph Theory. In this paper, we compute the multiplicative product connectivity Revan index, multiplicative sum connectivity Revan index, multiplicative ABC Revan index for polycyclic aromatic hydrocarbons and jagged rectangle benzenoid systems.
\end{abstract}

Keywords: Chemical graph, multiplicative product connectivity Revan index, multiplicative sum connectivity Revan index, multiplicative $A B C$ Revan index, polycyclic aromatic hydrocarbon, benzenoid system.

AMS Mathematics Subject Classification (2010): 05C05, 05C07, $05 C 90$

\section{Introduction}

We consider only finite, connected, simple graph $G$ with vertex set $V(G)$ and edge set $E(G)$. The degree $d_{G}(v)$ of a vertex $v$ is the number of vertices adjacent to $v$. Let $\Delta(G)(\delta(G))$ denote the maximum (minimum) degree among the vertices of $G$. The revan vertex degree $r_{G}(v)$ of a vertex $v$ in $G$ is defined as $r_{G}(v)=\Delta(G)+\delta(G)-d_{G}(v)$. The Revan edge connecting the Revan vertices $u$ and $v$ will be denoted by $u v$. For other undefined notations and terminology, the readers are referred to [1].

A topological index is the numeric quantity from the structural graph of a molecule. Topological indices have been found to be useful in chemical documentation, isomer discrimination, structure property relationships, structure activity relationships and pharmaceutical drug design in Organic chemistry. There has been considerable interest in the general problem of determining topological indices.

One of the best known topological index is the product connectivity Revan index, introduced by Kulli in [2]. The product connectivity Revan index of a graph $G$ is defined as

$$
P R(G)=\sum_{u v \in E(G)} \frac{1}{\sqrt{r_{G}(u) r_{G}(v)}} .
$$

Recently, Revan indices were studied, for example, in $[3,4,5,6]$. 
Motivated by the definition of the product connectivity Revan index, Kulli [7] introduced the multiplicative product connectivity Revan index, multiplicative sum connectivity Revan index and multiplicative atom bond connectivity Revan index of a graph as follows:

The multiplicative product connectivity Revan index of a graph $G$ is defined as

$$
\operatorname{PRII}(G)=\prod_{u v \in E(G)} \frac{1}{\sqrt{r_{G}(u) r_{G}(v)}}
$$

The multiplicative sum connectivity Revan index of a graph $G$ is defined as

$$
\operatorname{SRII}(G)=\prod_{u v \in E(G)} \frac{1}{\sqrt{r_{G}(u)+r_{G}(v)}}
$$

The multiplicative atom bond connectivity Revan index of a graph $G$ is defined as

$$
A B C R(G)=\prod_{u v \in E(G)} \sqrt{\frac{r_{G}(u)+r_{G}(v)-2}{r_{G}(u) r_{G}(v)}}
$$

Recently several multiplicative indices were studied, for example, in $[8,9,10$, $11,12,13,14,15,16$ to 18,19$]$. Also some connectivity indices were studied, for example, in $[20,21,22,23,24]$.

In this paper, we determine the multiplicative product connectivity Revan index, the multiplicative sum connectivity Revan index and the multiplicative atom bond connectivity index for polycyclic aromatic hydrocarbons $P A H_{n}$ and jagged rectangle benzenoid systems $B_{m, n}$. For more information about aromatic hydrocarbons and benzenoid systems see [25].

\section{Results for polycyclic aromatic hydrocarbons}

In this section, we focus on the chemical graph structure of the family of polycyclic aromatic hydrocarbons, denoted by $P A H_{n}$. The first three members of the family $P A H_{n}$ are given in Figure 1.

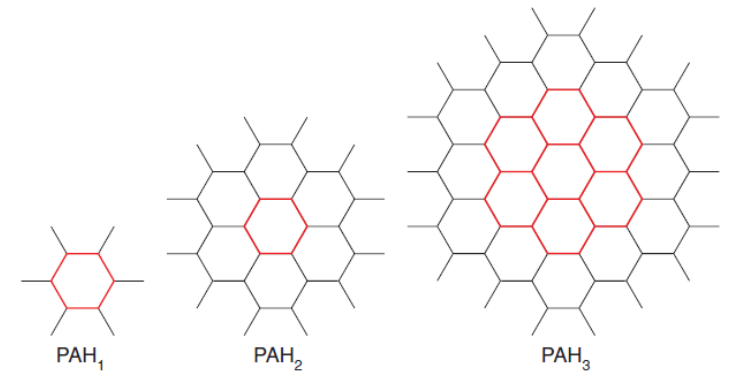

Figure 1:

In the following theorem, we compute the multiplicative product connectivity Revan index of $P A H_{n}$.

Theorem 1. Let $P A H_{n}$ be the family of polycyclic aromatic hydrocarbons. Then 
Multiplicative Connectivity Revan Indices of Polycyclic Aromatic Hydrocarbons and Benzenoid Systems

$$
\operatorname{PRII}\left(\text { PAH }_{n}\right)=\left(\frac{1}{3}\right)^{3 n} \text {. }
$$

Proof: Let $G=P A H_{n}$ be the chemical graph in the family of polycyclic aromatic hydrocarbons. We see that the vertices of $G$ are either of degree 1 or 3 . Therefore $\Delta(G)=3$ and $\delta(G)=1$. Thus $r_{G}(u)=\Delta(G)+\delta(G)-d_{G}(u)=4-d_{G}(u)$. By calculation, we obtain that $G$ has $6 n^{2}+6 n$ vertices and $9 n^{2}+3 n$ edges, see [25]. In $G$, there are two types of edges based on the degree of end vertices of each edge as follows:

$$
\begin{array}{ll}
E_{13}=\left\{u v \in E(G) \mid d_{G}(u)=1, d_{G}(v)=3\right\}, & \left|E_{13}\right|=6 n . \\
E_{33}=\left\{u v \in E(G) \mid d_{G}(u)=d_{G}(v)=3\right\}, & \left|E_{33}\right|=9 n^{2}-3 n .
\end{array}
$$

Thus there are two types of Revan edges based on the revan degree of end revan vertices of each revan edge as follows:

$$
\begin{array}{llrl}
R E_{31} & =\left\{u v \in E(G) \mid r_{G}(u)=3, r_{G}(v)=1\right\}, & & \left|R E_{31}\right|=6 n . \\
R E_{11} & =\left\{u v \in E(G) \mid r_{G}(u)=r_{G}(v)=1\right\}, & & \left|R E_{11}\right|=9 n^{2}-3 n .
\end{array}
$$

To compute $P R I I\left(P A H_{n}\right)$, we see that

$$
\begin{aligned}
\operatorname{PRII}\left(\text { PAH }_{n}\right) & =\prod_{u v \in E(G)} \frac{1}{\sqrt{r_{G}(u) r_{G}(v)}} \\
& =\prod_{R E_{31}} \frac{1}{\sqrt{r_{G}(u) r_{G}(v)}} \times \prod_{R E_{11}} \frac{1}{\sqrt{r_{G}(u) r_{G}(v)}} \\
& =\left(\frac{1}{\sqrt{3 \times 1}}\right)^{6 n} \times\left(\frac{1}{\sqrt{1 \times 1}}\right)^{9 n^{2}-6 n}=\left(\frac{1}{3}\right)^{3 n} .
\end{aligned}
$$

In the next theorem, we compute the multiplicative sum connectivity Revan index of $P A H_{n}$.

Theorem 2. Let $P A H_{n}$ be the family of polycyclic aromatic hydrocarbons. Then

$$
\operatorname{SRII}\left(P_{A} H_{n}\right)=\left(\frac{1}{\sqrt{2}}\right)^{9 n(n+1)} .
$$

Proof: Let $\mathrm{G}=P A H_{n}$ be the chemical graph in the family of polycyclic aromatic hydrocarbons. From equation (2) and by cardinalities of the revan edge partition of $P A H_{n}$ polycyclic aromatic hydrocarbons, we have

$$
\begin{aligned}
\operatorname{SRII}\left(P A H_{n}\right) & =\prod_{u v \in E(G)} \frac{1}{\sqrt{r_{G}(u)+r_{G}(v)}} \\
& =\prod_{R E_{31}} \frac{1}{\sqrt{r_{G}(u)+r_{G}(v)}} \times \prod_{R E_{11}} \frac{1}{\sqrt{r_{G}(u)+r_{G}(v)}} \\
& =\left(\frac{1}{\sqrt{3+1}}\right)^{6 n} \times\left(\frac{1}{\sqrt{1+1}}\right)^{9 n^{2}-3 n}=\left(\frac{1}{\sqrt{2}}\right)^{9 n(n+1)} .
\end{aligned}
$$


In the following theorem, we compute the multiplicative atom bond connectivity Revan index of $P A H_{n}$.

Theorem 3. Let $P A H_{n}$ be the family of polycyclic aromatic hydrocarbons. Then

$$
A B C R I I\left(P A H_{n}\right)=0 \text {. }
$$

Proof: Let $G=P A H_{n}$ be the chemical graph in the family of polycyclic aromatic hydrocarbons, from equation (3) and by cardinalities of the revan edge partition of $P A H_{n}$ polycyclic aromatic hydrocarbons, we have

$$
\begin{aligned}
\operatorname{ABCRII}\left(P A H_{n}\right) & =\prod_{u v \in E(G)} \sqrt{\frac{r_{G}(u)+r_{G}(v)-2}{r_{G}(u) r_{G}(v)}} \\
& =\prod_{R E_{31}} \sqrt{\frac{r_{G}(u)+r_{G}(v)-2}{r_{G}(u) r_{G}(v)} \times \prod_{R E_{11}} \sqrt{\frac{r_{G}(u)+r_{G}(v)-2}{r_{G}(u) r_{G}(v)}}} \\
& =\left(\sqrt{\frac{3+1-2}{3 \times 1}}\right)^{6 n} \times\left(\sqrt{\frac{1+1-2}{1 \times 1}}\right)^{9 n^{2}-3 n}=0 .
\end{aligned}
$$

\section{Results for benzenoid systems}

In this section, we focus on the chemical graph structure of a jagged rectangle benzenoid system, denoted by $B_{m, n}$ for all $m, n \in N$. Three chemical graphs of a jagged rectangle benzenoid systems are shown in Figure 2.
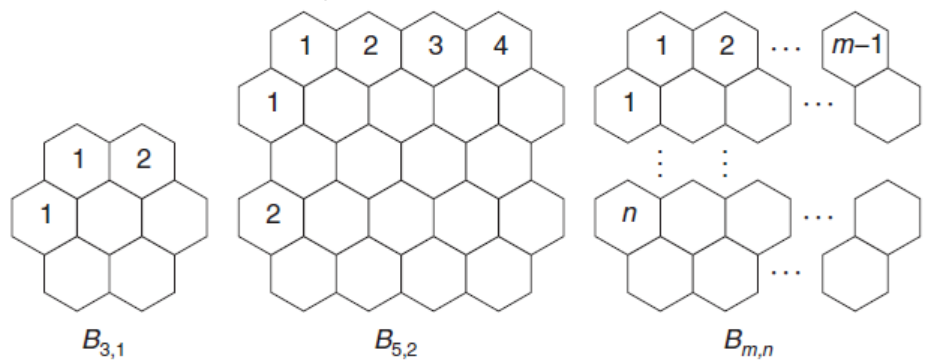

Figure 2:

In the following theorem, we compute the multiplicative product connectivity Revan index of $B_{m, n}$.

Theorem 4. Let $B_{m, n}$ be the family of a jagged rectangle system. Then

$$
\operatorname{PRII}\left(B_{m, n}\right)=\left(\frac{1}{3}\right)^{2 n+4} \times\left(\frac{1}{6}\right)^{2 m+2 n-2} \times\left(\frac{1}{2}\right)^{6 m n+m-5 n-4} .
$$

Proof: Let $G=B_{m, n}$ be the chemical graph in the family of a jagged rectangle benzenoid system. We see that the vertices of $G$ are either of degree 2 or 3 . Therefore $\Delta(G)=3$ and $\delta(G)=2$. Thus $r_{G}(u)=\Delta(G)+\delta(G)-d_{G}(u)=5-d_{G}(u)$. By calculation, we obtain that $G$ has $4 m n+4 m+2 n-2$ vertices and $6 m n+5 m+n-4$ edges, see [25]. In $G$, there are three types of edges based on the degree of end vertices of each edge as follows: 
Multiplicative Connectivity Revan Indices of Polycyclic Aromatic Hydrocarbons and Benzenoid Systems

$$
\begin{array}{llrl}
E_{22}=\left\{u v \in E(G) \mid d_{G}(u)=d_{G}(v)=2\right\}, & & \left|E_{22}\right|=2 n+4 . \\
E_{23}=\left\{u v \in E(G) \mid d_{G}(u)=2, d_{G}(v)=3\right\}, & & \left|E_{23}\right|=4 m+4 n-4 . \\
E_{33}=\left\{u v \in E(G) \mid d_{G}(u)=d_{G}(v)=3\right\}, & & \left|E_{33}\right|=6 m n+m-5 n-4 .
\end{array}
$$

Thus there are three types of Revan edges based on the revan degree of end revan vertices of each revan edge as follows:

$$
\begin{array}{rlrl}
R E_{33}=\left\{u v \in E(G) \mid r_{G}(u)=r_{G}(u)=3\right\}, & & \left|R E_{33}\right|=2 n+4 . \\
R E_{32}=\left\{u v \in E(G) \mid r_{G}(u)=3, r_{G}(u)=2\right\}, & & \left|R E_{32}\right|=4 m+4 n-4 . \\
R E_{22}=\left\{u v \in E(G) \mid r_{G}(u)=r_{G}(u)=2\right\}, & & \left|R E_{22}\right|=6 m n+m-5 n-4 . \\
\text { To compute } P R I I\left(B_{m, n}\right), \text { we see that } & \\
\operatorname{PRII}\left(B_{m, n}\right)= & \prod_{u v E(G)} \frac{1}{\sqrt{r_{G}(u) r_{G}(v)}} \\
& =\prod_{R E_{33}} \frac{1}{\sqrt{r_{G}(u) r_{G}(v)}} \times \prod_{R E_{32}} \frac{1}{\sqrt{r_{G}(u) r_{G}(v)}} \times \prod_{R E_{22}} \frac{1}{\sqrt{r_{G}(u) r_{G}(v)}} \\
& =\left(\frac{1}{\sqrt{3 \times 3}}\right)^{2 n+4} \times\left(\frac{1}{\sqrt{3 \times 2}}\right)^{4 m+4 n-4} \times\left(\frac{1}{\sqrt{2 \times 2}}\right)^{6 m n+m-5 n-4} \\
& =\left(\frac{1}{3}\right)^{2 n+4} \times\left(\frac{1}{6}\right)^{2 m+2 n-2} \times\left(\frac{1}{2}\right)^{6 m n+m-5 n-4}
\end{array}
$$

In the following theorem, we compute the multiplicative sum connectivity Revan index of $B_{m, n}$.

Theorem 5. Let $B_{m, n}$ be the family of a jagged rectangle benzenoid system. Then

$$
\operatorname{SRII}\left(B_{m, n}\right)=\left(\frac{1}{6}\right)^{n+2} \times\left(\frac{1}{5}\right)^{2 m+2 n-2} \times\left(\frac{1}{2}\right)^{6 m n+m-5 n-4} .
$$

Proof: Let $G=B_{m, n}$ be the chemical graph in the family of a jagged rectangle benzenoid system. From equation (2) and by the cardinalities of the revan edge partition of $B_{m, n}$, we have

$$
\begin{aligned}
\operatorname{SRII}\left(B_{m, n}\right) & =\prod_{u v E(G)} \frac{1}{\sqrt{r_{G}(u)+r_{G}(v)}} \\
& =\prod_{R E_{33}} \frac{1}{\sqrt{r_{G}(u)+r_{G}(v)}} \times \prod_{R E_{32}} \frac{1}{\sqrt{r_{G}(u)+r_{G}(v)}} \times \prod_{R E_{22}} \frac{1}{\sqrt{r_{G}(u)+r_{G}(v)}} \\
& =\left(\frac{1}{\sqrt{3+3}}\right)^{2 n+4} \times\left(\frac{1}{\sqrt{3+2}}\right)^{4 m+4 n-4} \times\left(\frac{1}{\sqrt{2+2}}\right)^{6 m n+m-5 n-4} \\
& =\left(\frac{1}{6}\right)^{n+2} \times\left(\frac{1}{5}\right)^{2 m+2 n-2} \times\left(\frac{1}{2}\right)^{6 m n+m-5 n-4} .
\end{aligned}
$$


In the next theorem, we compute the multiplicative atom bond connectivity Revan index of $B_{m, n}$.

Theorem 6. Let $B_{m, n}$, be the family of a jagged rectangle benzenoid system. Then

$$
A B C R I I\left(B_{m, n}\right)=\left(\frac{2}{3}\right)^{2 n+4} \times\left(\frac{1}{\sqrt{2}}\right)^{6 m n+5 m-n-8} .
$$

Proof: Let $G=B_{m, n}$ be a chemical graph in the family of a jagged rectangle benzenoid system. From equation (3) and by cardinalities of the revan edge partition of $B_{m, n}$, we have

$$
\begin{aligned}
\operatorname{ABCRII} & \left(B_{m, n}\right)=\prod_{u v \in E(G)} \sqrt{\frac{r_{G}(u)+r_{G}(v)-2}{r_{G}(u) r_{G}(v)}} \\
& =\left(\sqrt{\frac{3+3-2}{3 \times 3}}\right)^{2 n+4} \times\left(\sqrt{\frac{3+2-2}{3 \times 2}}\right)^{4 m+4 n-4} \times\left(\sqrt{\frac{2+2-2}{2 \times 2}}\right)^{6 m n+m-5 n-4} \\
& =\left(\frac{2}{3}\right)^{2 n+4} \times\left(\frac{1}{\sqrt{2}}\right)^{6 m n+5 m-n-8}
\end{aligned}
$$

\section{REFERENCES}

1. V.R.Kulli, College Graph Theory, Vishwa International Publications, Gulbarga, India (2010).

2. V.R.Kulli, On the product connectivity Revan index of certain nanotubes, Journal of Computer and Mathematical Sciences, 8(10) (2017) 562-567.

3. V.R.Kulli, Revan indices of oxide and honeycomb networks, International Journal of Mathematics and its Applications, 5(4-E) (2017) 663-667.

4. V.R.Kulli, The sum connectivity Revan index of silicate and hexagonal networks, Annals of Pure and Applied Mathematics, 14(3) (2017) 401-406. DOI: http://dx.doi.org/10.22457/apam.v14n3a6.

5. V.R.Kulli, Multiplicative Revan and multiplicative hyper-Revan indices of certain networks, Journal of Computer and Mathematical Sciences, 8(12) (2017) 750-757.

6. V.R.Kulli, Revan indices and their polynomials of certain rhombus networks, submitted.

7. V.R.Kulli, Multiplicative connectivity Revan indices of certain families of benzenoid systems, submitted.

8. V.R.Kulli, Multiplicative connectivity indices of certain nanotubes, Annals of Pure and Applied Mathematics, 12(2) (2016) 169-176.

9. V.R.Kulli, General multiplicative Zagreb indices of $T U C_{4} C_{8}[m, n]$ and $T U C_{4}[m, n]$ nanotubes, International Journal of Fuzzy Mathematical Archive, 11(1) (2016) 3943.

10. V.R.Kulli, Multiplicative connectivity indices of $T U C_{4} C_{8}[m, n]$ and $T U C_{4}[m, n]$ nanotubes, Journal of Computer and Mathematical Sciences, 7(11) (2016) 599-605 
Multiplicative Connectivity Revan Indices of Polycyclic Aromatic Hydrocarbons and Benzenoid Systems

11. V.R.Kulli, On multiplicative $K$-Banhatti and multiplicative $K$ hyper-Banhatti indices of $V$-Phenylenic nanotubes and nanotorus, Annals of Pure and Applied Mathematics, 11(2) (2016) 145-150.

12. V.R.Kulli, Multiplicative $K$ hyper-Banhatti indices and coindices of graphs, International Journal of Mathematical Archive, 7(6) (2016) 60-65.

13. V.R.Kulli, Two new multiplicative atom bond connectivity indices, Annals of Pure and Applied Mathematics, 13(1) (2017) 1-7.

DOI: http://dx.doi.org/10.22457/apam.vl3nlal.

14. V.R.Kulli, Multiplicative connectivity indices of nanostructures, Journal of Ultra Scientist of Physical Sciences, A 29(1) (2017) 1-10.

DOI: http://dx.doi-org/10.22147/jusps.A/290101.

15. V.R.Kulli, A new multiplicative arithmetic-geometric index, International Journal of Fuzzy Mathematical Archive, 12(2) (2017) 49-53. DOI:http://dx.doi.org/10.22457/ijfma.v12n2a1.

16. V.R.Kulli, Some new multiplicative geometric-arithmetic indices, Journal of Ultra Scientist of Physical Sciencs, A, 29(2) (2017) 52-57. DOI: http://dx.doi.org/10.22147/jusps.A/290201.

17. V.R.Kulli, New multiplicative inverse sum indeg index of certain benzoid systems, Journal of Global Research in Mathematical Achieves, 4(10) (2017) 15-19.

18. V.R.Kulli, New multiplicative arithmetic-geometric indices, Journal of Ultra Scientist of Physical Sciences, A, 29(6) (2017) 205-211. DOI: http://dx.doi.org/10.22147/jusps-A/290601.

19. V.R.Kulli, Edge version of multiplicative connectivity indices of some nanotubes and nanotorus, International Journal of Current Research in Science and Technology, 3(11) (2017) 7-15.

20. V.R.Kulli, On the product connectivity reverse index of silicate and hexagonal networks, International Journal of Mathematics and its Applications, 5(4-B) (2017) 175-179.

21. V.R.Kulli, On the sum connectivity reverse index of oxide and honeycomb networks, Journal of Computer and Mathematical Sciences, 8(9) (2017) 408-413.

22. V.R.Kulli, The product connectivity Gourava index, Journal of Computer and Mathematical Sciences, 8(6) (2017) 235-242.

23. V.R.Kulli, On the sum connectivity Gourava index, International Journal of Mathematical Archive, 8(7)(2017) 211-217.

24. V.R.Kulli, B.Chaluvaraju and H.S.Boregowda, Some degree based connectivity indices of Kulli cycle windmill graphs, South Asian Journal of Mathematics, 6(6) (2016) 263-268.

25. V.R.Kulli, B.Stone, S.Wang and B.Wei, Generalized multiplicative indices of polycyclic aromatic hydrocarbons and benzenoid systems, Z. Naturforsch, 72(6) (2017) 573-576. 